\title{
A Vitamin D, Calcium and Leucine-Enriched Whey Protein Nutritional Supplement Improves Measures of Bone Health in Sarcopenic Non-Malnourished Older Adults: The PROVIDE Study
}

\author{
Tom R. Hill ${ }^{10}$. Sjors Verlaan ${ }^{2,5}$ - Egbert Biesheuvel ${ }^{2} \cdot$ Richard Eastell $^{3} \cdot$ Jürgen M. Bauer $^{6} \cdot$ Ivan Bautmans $^{7}$. \\ Kirsten Brandt ${ }^{1}$ - Lorenzo M. Donini ${ }^{8} \cdot$ Marcello Maggio $^{9} \cdot$ Tony Mets $^{7} \cdot$ Chris J. Seal $^{1}$. Sander LJ Wijers ${ }^{2}$. \\ Cornel Sieber ${ }^{10} \cdot$ Tommy Cederholm $^{11} \cdot$ Terry J. Aspray $^{4}$ on behalf of the PROVIDE Consortium
}

Received: 28 November 2018 / Accepted: 2 July 2019 / Published online: 23 July 2019

(c) The Author(s) 2019

\begin{abstract}
Alterations in musculoskeletal health with advanced age contribute to sarcopenia and decline in bone mineral density (BMD) and bone strength. This decline may be modifiable via dietary supplementation. To test the hypothesis that a specific oral nutritional supplement can result in improvements in measures of bone health. Participants ( $n$ 380) were participants of the PROVIDE study, a 13-week, multicenter, randomized, controlled, double-blind, 2 parallel-group study among nonmalnourished older participants ( $\geq 65$ years) with sarcopenia [determined by Short Physical Performance Battery (SPPB; 0 -12) scores between 4 and 9, and a low skeletal muscle mass index (SMI; skeletal muscle mass/BW $\times 100$ ) $\leq 37 \%$ in men and $\leq 28 \%$ in women using bioelectric impedance analysis] Supplementation of a vitamin D, calcium and leucine-enriched whey protein drink that comprises a full range of micronutrients (active; $2 /$ day) was compared with an iso-caloric control. Serum 25-hydroxyvitamin D [25(OH)D], parathyroid hormone (PTH), biochemical markers of bone formation (osteocalcin; OC, procollagen type 1 amino-terminal propeptide; P1NP) and resorption (carboxy-terminal collagen crosslinks; CTX), insulin like growth factor 1 (IGF-1) and total-body BMD were analysed pre- and post-intervention. Serum $25(\mathrm{OH})$ $\mathrm{D}$ concentrations increased from $51.1 \pm 22.9 \mathrm{nmol} / \mathrm{L}($ mean $\pm \mathrm{SD})$ to $78.9 \pm 21.1 \mathrm{nmol} / \mathrm{L}$ in the active group $(p<0.001 \mathrm{vs}$. control). Serum PTH showed a significant treatment difference $(p<0.001)$ with a decline in the active group, and increase in the control group. Serum IGF-1 increased in the active group ( $p<0.001$ vs. control). Serum CTX showed a greater decline in the active group ( $p=0.001$ vs. control). There were no significant differences in serum OC or P1NP between groups during the intervention. Total body BMD showed a small $\left(0.02 \mathrm{~g} / \mathrm{cm}^{2} ; \sim 2 \%\right)$ but significant increase in the active group after supplementation ( $p=0.033$ vs. control). Consuming a vitamin $\mathrm{D}$, calcium and leucine-enriched whey protein supplement for 13 weeks improved 25(OH)D, suppressed PTH and had small but positive effects on BMD, indicative of improved bone health, in sarcopenic non-malnourished older adults.
\end{abstract}

Keywords Vitamin D $\cdot$ Leucine $\cdot$ Intervention $\cdot$ Bone turnover $\cdot$ BMD $\cdot$ PROVIDE Study

\section{Introduction}

Loss of muscle mass and function (i.e. sarcopenia), and decline in bone mineral density (BMD) and bone strength (i.e. osteopenia and osteoporosis) lead to alterations in musculoskeletal health with advanced age [1]. Both disorders increase the risk of fractures, loss of independence, and

Tom R. Hill

Tom.hill@ncl.ac.uk

Extended author information available on the last page of the article mortality [2] but the risk is highest in those with so-called osteosarcopenia where both disorders coincide [3]. A bilateral cross-talk between muscle and bone has been suggested [4], with underlying causal factors that partly overlap.

Like muscle, bone is a dynamic tissue that responds to the external and internal environments to which it is exposed during an individual's lifetime. While a considerable variations in bone mass is considered genetically determined (up to $70 \%$ ), lifestyle factors such as nutrition and exercise are well-established modifiable factors of bone mass. Of the modifiable nutritional factors, dietary protein, vitamin D and calcium are well known [5]. In a 
study of 82 patients (mean age, 80.7 years) with recent osteoporotic hip fracture, protein deficiency was associated with accelerated bone resorption and impaired bone formation [6]. Adequate protein intake increases insulinlike growth factor 1 (IGF-1) and promotes calcium absorption, potentially slowing bone loss [7]. Garnero et al. reported an association between increased fracture risk and low-IGF-1 levels, independent of BMD, in healthy postmenopausal women [8]. A recent meta-analysis in adults ( $\geq 18$ years) showed positive trends of higher protein intake (defined as either $>90 \mathrm{~g}$ protein/day, $25 \%$ and $30 \%$ of total energy from protein/day or $1.4 \mathrm{~g}_{\text {protein }} \mathrm{kg}^{-1} /$ day) on lumber spine BMD [9]. Apart from the absolute protein intake level, there is some evidence that the intake of specific amino acids is associated with higher spine and forearm BMD, with the strongest association observed for leucine [10]. Moreover, dietary essential amino acid supplementation in rats on a low-protein diet increased bone strength through significant (2-4\%) increases in lumbar spine and tibia BMD, trabecular architecture, and cortical thickness, likely mediated via an increase of IGF-1 [11]. This is also reflected by the dietary recommendation for prevention of age-related deterioration of musculoskeletal health in postmenopausal women by The European Society for Clinical and Economic Aspects of Osteoporosis and Osteoarthritis (ESCEO). The ESCEO recommends a daily protein intake of $1.0-1.2 \mathrm{~g} / \mathrm{kg}$ with at least 20 to $25 \mathrm{~g}$ of high-quality protein at each main meal, adequate vitamin D intake at $800 \mathrm{IU} /$ day to maintain serum $25(\mathrm{OH})$ $\mathrm{D}$ above $50 \mathrm{nmol} / \mathrm{L}$ as well as calcium intake of $1000 \mathrm{mg} /$ day. These recommendations for protein and vitamin $\mathrm{D}$ are in line with those from the PRO-TAGE study group [12] and Institute of Medicine [13] for older adults.

In a previous publication [14], we described the intervention effects of a targeted nutritional supplement containing whey protein enriched with leucine, vitamin $\mathrm{D}$, calcium and a full range of micronutrients on macronutrient intake and muscle mass and lower-extremity function in older sarcopenic adults. A nutritional intervention can improve the functional capacity in older adults with an inadequate nutritional status. To establish an (detectable) increase in muscle mass, in addition to improvement of muscle quality, a sufficient $25(\mathrm{OH}) \mathrm{D}$ status and protein intake may be required as we have previously shown [15]. Therefore, we hypothesised that providing the same nutritional intervention in sarcopenic older adults would improve markers of musculoskeletal health. Here, we report the efficacy of this supplement for improving serum 25(OH)D and reducing parathyroid hormone (PTH) as well as altering biochemical markers of bone formation (Osteocalcin; OC, Procollagen type 1 amino-terminal propeptide; P1NP), resorption (carboxy-terminal collagen crosslinks; CTX), serum IGF-1 and total body BMD.

\section{Methods}

\section{Design and Participants}

Participants were members of the PROVIDE study, a 13-week, multicenter, randomized, controlled, doubleblind, two parallel-group study among non-malnourished older participants ( $>65$ years) with mobility limitations and reduced muscle mass [14]. The study protocol, approvals and participant characteristics have been described in detail elsewhere [14] but in brief, participants were recruited from 18 study centres in 6 European countries: Belgium, Germany, Ireland, Italy, Sweden, and the United Kingdom. Participants were screened for mild to moderate limitations in physical function (SPPB score 4-9), and for low-skeletal Muscle Mass Index [SMI; (skeletal muscle mass $/ \mathrm{BW} \times 100) \leq 37 \%$ in men and $\leq 28 \%$ in women] [1] using bioelectric impedance analysis (BIA 101; Akern, Florence, Italy) at each location [16]. Based on the work by Sergi et al. [17], the BIA method reports a $1 \%$ precision for resistance $(\mathrm{Rz})$ and $5 \%$ precision for reactance (Xc). Furthermore, participants were eligible to participate if they had a body mass index (BMI) between 20 and $30 \mathrm{~kg} / \mathrm{m}^{2}$, no major cognitive impairment [Mini Mental State Examination (MMSE) score $\geq 25$ ], and were able and willing to provide informed consent. Potential participants were excluded if they had comorbidities such as kidney or liver failure, malignancies over the past 5 years, anemia, or acute inflammation (C-reactive protein concentration $>10 \mathrm{mg} / \mathrm{L}$ ), presented with contraindications for calcium/vitamin D supplementation and/or were using medication interfering with the nutritional intervention. Users of protein containing or amino acid-containing nutritional supplements 3 months before starting and during the study were excluded. Moreover, throughout the study, participants were instructed not to change nutritional habits, including nutritional supplements. With regard to medical use of vitamin D and calcium intake from medical sources, the following exclusion criteria applied:

- More than $22 \mu \mathrm{g}$ (880 IU) of daily vitamin D intake from medical sources.

- Between $11.25 \mu \mathrm{g}$ (450 IU) and $22 \mu \mathrm{g}$ (880 IU) of daily vitamin D intake from medical sources in combination with a serum calcidiol $(25(\mathrm{OH}) \mathrm{D}$; vitamin $\mathrm{D})$ level $\geq 50 \mathrm{nmol} / \mathrm{L}$.

- More than $500 \mathrm{mg}$ of daily calcium intake from medical sources.

A diagnosis of osteoporosis and use of anti-osteoporotic medications were not defined as exclusion criteria 
and osteoporosis was not assessed from the DXA scans. Medical history for known osteoporosis at baseline indicates that $10.3 \%$ of participants were osteoporotic, with no difference between the control and treatment groups. Further details on medical visits/falls, dietary assessment, the MMSE, Geriatric Depression Score (GDS), Physical Activity Scale for the Elderly (PASE), and assessment of appendicular muscle mass have been described previously [14].

Participants were randomized by permuted block randomization (block size 4) to the active or control group was stratified for SPPB categories 4 to 6 and 7 to 9 and study center [14]. The randomization sequence was computergenerated by a blinded statistician not involved in data collection or analysis. All investigators, study staff, and participants were blinded to group allocations, and the randomization code was not broken until statistical modelling of the primary and secondary outcomes was complete. The active product contained, per serving, $20 \mathrm{~g}$ of whey protein, $3 \mathrm{~g}$ of total leucine, $9 \mathrm{~g}$ of carbohydrates, $3 \mathrm{~g}$ of fat, $800 \mathrm{IU}$ of vitamin D, $500 \mathrm{mg}$ of calcium and a mixture of vitamins, minerals, and fibers, whereas the iso-caloric control product did not contain any protein or micronutrients, and only carbohydrates, fat, and some trace elements [14]. Both were delivered as $40 \mathrm{~g}$ sachets with powder to be reconstituted with 100 to $150 \mathrm{~mL}$ water and consumed twice daily before breakfast and lunch to provide an adequate bolus of protein with these meals [14]. A more even distribution of protein ingestion throughout the day, providing adequate amounts of protein (25-30 g) per meal, has been suggested to modify net muscle protein balance on a 24-h basis [18]. Since breakfast and lunch are the meals that in general do not meet the 25-30 g protein guideline, participants were advised to take the supplement at breakfast and lunch. Study products were provided by Nutricia Research, Nutricia Advanced Medical Nutrition. Product compliance was measured using selfcompleted intake diaries. Adequate compliance was defined as having consumed 10 of the possible 14 servings per week. Dietary assessment was conducted at baseline and week 13 using 3-days prospective diet records for 2 week-days and 1 weekend day. Additional energy and protein intakes from both supplements were added to the habitual 3-day intakes (i.e., non-supplementary intake) to assess total intakes. Food records were checked for completeness with participants during study visits and additional information was obtained about unclear items or amounts. Total energy, macronutrient and micronutrient intakes were calculated by the participating sites using site- and country-specific dietary data entry systems and food composition tables [14].

The study protocol was reviewed and approved by The Research Ethics Boards at each of the locations and was registered in the Dutch trials register with the identifier: NTR2329 (http://www.trialregister.nl/trialreg).

\section{Outcome Measures}

Standardized total body BMD measurement was performed by dual-energy X-ray absorptiometry (DXA; different models from Hologic, Bedford, MA and Lunar, Fairfield, CT). Daily calibration of the densitometers was performed in accordance with the instructions provided by the manufacturer. The scanning procedure was standardised across all study centres using a uniform protocol. All raw DXA data were centrally analysed by the same experienced investigator who was blinded for group allocation and study outcomes. For Hologic, the intramachine CV for BMD is $1.6 \%$; the interrater CV is $1.8 \%$ [19].

Serum samples were processed from whole blood as previously described $[14,15]$. All serum samples were analysed in the same lab but in several batches. Analytical testing for total serum vitamin $\mathrm{D}[25(\mathrm{OH}) \mathrm{D}]$ was performed using chemiluminescense micro-particulate immunoassay (Abbott Laboratories, Wiesbaden, Germany). Serum 25(OH) D concentrations were considered suboptimal if they were below $50 \mathrm{nmol} / \mathrm{L}$ [13]. Serum intact PTH concentrations were measured in serum using an ELISA (Intact parathyroid hormone, MD Biosciences Inc., St. Paul, MN 55108), with an intra-assay CV of 3.4\%. Total calcium and albumin concentrations in serum were measured by an automated system (Instrumentation Laboratories UK Ltd, Cheshire, UK). Serum calcium concentrations were adjusted for albumin concentration. The intra-assay $\mathrm{CV}$ for serum calcium and albumin was $1.7 \%$ and $1.8 \%$, respectively. OC levels were measured in serum samples using an ELISA (Metra ${ }^{\mathrm{TM}}$ Osteocalcin EIA Kit, Quidel Corporation, CA, USA), with an intra-assay CV of $6.0 \%$. Bone turnover markers CTX and P1NP were measured using the Cobas e411 automated electrochemiluminescent immunoassay (ECLIA) (Roche Diagnostics, Penzberg, Germany). The inter assay coefficients of variation (CVs) were $<5 \%$. IGF-1 was also measured in serum samples using an ELISA. IGF-1 was assessed with a CLIA Immulite kit L2KGF2 (Siemens Healthcare Diagnostics, Upplands Vôssby, Sweden). Analysis was performed on Immulite 200-Xpi instrument and the inter- and intra-assay variation was $3.9 \%$ and $7.4 \%$, respectively.

\section{Statistical Analyses}

Analyses were performed as intention-to-treat, defined as all participants randomized, regardless of whether they finished the full study protocol. Descriptive statistics [mean and SD, median and interquartile range (IQR), where appropriate] were determined for all variables. Variables were log- or square root-transformed to achieve near normal distributions where appropriate. Baseline characteristics of participants in the two intervention groups were compared using Fisher's exact test (for categorical variables) or two-sample 
t-tests or Mann-Whitney $U$ test for continuous variables. The change from pre- to post-intervention values for the biochemical measures and BMD was compared between the active and control group using a two-sample $t$ test. In addition, ANCOVA was used to test for the effect of the treatment on post-intervention variables while controlling for pre-intervention concentrations and covariates age, sex and study site. All tests were two-sided with a significance level of 5\%. All statistical analyses were done using SAS software (version 9.4; SAS, Inc, Cary, NC).

\section{Results}

Between June 30, 2010, and May 30, 2013, 1240 older adults were screened for participation, 380 of whom were randomized to the active or control groups. After the 13-week intervention, 302 participants completed all three study visits (79\% completion rate). The reason for drop-out of 78 study participants are: adverse events $(n=45)$, a serious adverse event ( $n=2$; not related to product intake), withdrawal of informed consent $(n=15)$, lost to follow up $(n=2)$, protocol deviation (participant taking calcium supplements), another reason $(n=13)$ [14]. During the RCT there was no significant difference in the number of adverse events between the test group (45 subjects; $25.0 \%$ ) and the control group (54 subjects; $27.7 \%)(p=0.562)$. The most commonly reported related adverse events were gastro-intestinal (such as abdominal pain and nausea) or metabolic and nutritional disorders (such as hyperglycaemia). No treatment-related serious adverse events occurred during the RCT. The mean age of the population at enrolment was 77.7 years $(65 \%$ women) and living independently (87\%). Baseline characteristics were similar in both groups (Table 1). At baseline, the $\%$ of participants with $25(\mathrm{OH}) \mathrm{D}$ concentrations below $50 \mathrm{nmol} / \mathrm{L}$ was $51 \%$ and $53 \%$ in the control and active group, respectively $(p>0.05)$. All participants had low muscle mass, a mean SPPB score of 7.5, a mean BMI of $26.1 \mathrm{~kg}$ / $\mathrm{m}^{2}$, and were non-malnourished based on the Mini Nutritional Assessment Short-Form (99.5\%). Ten per cent of the

Table 1 Descriptive statistics comparing history, body composition, clinical outcomes and dietary intake at baseline between adults on control and active treatment

\begin{tabular}{|c|c|c|c|c|}
\hline & & Statistic & Control $n=196$ & Active $n=184$ \\
\hline Sex, female* & & $\mathrm{n}(\%)$ & $129(65.8)$ & $120(65.2)$ \\
\hline Age at screening (years) ${ }^{\mathrm{a}}$ & & Mean (SD) & $78.1(7.0)$ & $77.3(6.7)$ \\
\hline \multirow[t]{3}{*}{ MNA categories, screening score ${ }^{b}$} & 0-7 points (malnutrition) & $\mathrm{n}(\%)$ & $1(0.5)$ & $1(0.5)$ \\
\hline & $\begin{array}{l}8-11 \text { points (risk of malnu- } \\
\text { trition) }\end{array}$ & $\mathrm{n}(\%)$ & $19(9.7)$ & $15(8.2)$ \\
\hline & $\begin{array}{l}12-14 \text { points (no malnutri- } \\
\text { tion) }\end{array}$ & $\mathrm{n}(\%)$ & $176(89.8)$ & $168(91.3)$ \\
\hline $\begin{array}{l}\text { Subjects with medical or hospital visit } 3 \text { months } \\
\text { before the study* }\end{array}$ & & $\mathrm{n}(\%)$ & $164(83.7)$ & $151(82.1)$ \\
\hline Subjects reporting falls 3 months before the study* & & $\mathrm{n}(\%)$ & $180(91.8)$ & $170(92.4)$ \\
\hline Geriatric Depression Scale ${ }^{\mathrm{b}}$ & & Median (IQR) & $2.0(1.0,3.0)$ & $2.0(1.0,3.0)$ \\
\hline $\operatorname{MMSE}^{\mathrm{b}}$ & & Median (IQR) & $29.0(28.0,30.0)$ & $29.0(27.0,30.0)$ \\
\hline PASE questionnaire $^{\mathrm{a}}$ & & Mean (SD) & $98.0(73.2)$ & $101.6(71.0)$ \\
\hline Weight, $\mathrm{kg}^{\mathrm{a}}$ & & Mean (SD) & $69.5(10.9)$ & $70.2(11.6)$ \\
\hline Height, $\mathrm{m}^{\mathrm{a}}$ & & Mean (SD) & $1.63(0.09)$ & $1.64(0.10)$ \\
\hline BMI, $\mathrm{kg} / \mathrm{m}^{2 \mathrm{a}}$ & & Mean (SD) & $26.2(2.8)$ & $26.0(2.5)$ \\
\hline Appendicular muscle mass, $\mathrm{kg}, \mathrm{DXA}^{\mathrm{a}}$ & & Mean (SD) & $17.5(3.8)$ & $17.9(4.1)$ \\
\hline Appendicular muscle mass $/ \mathrm{h}^{2}, \mathrm{~kg} / \mathrm{m}^{2}, \mathrm{DXA}^{\mathrm{a}}$ & & Mean (SD) & $6.6(1.0)$ & $6.6(0.9)$ \\
\hline Dietary energy intake $(\mathrm{kcal} / \mathrm{day})^{\mathrm{a}}$ & & Mean (SD) & $1667.1(401.3)$ & $1756.7(456.3)$ \\
\hline Protein intake $(\mathrm{g} / \text { day })^{\mathrm{b}}$ & & Median (IQR) & $67(56,78)$ & $70(58,83)$ \\
\hline Protein intake $(\mathrm{g} / \mathrm{kg} / \mathrm{day})^{\mathrm{b}}$ & & Median (IQR) & $1.0(0.8,1.2)$ & $1.0(0.9,1.2)$ \\
\hline Protein intake, energy $\%^{\mathrm{b}}$ & & Median (IQR) & $17(15,19)$ & $16(15,19)$ \\
\hline Vitamin D intake $(\mu \mathrm{g})^{\mathrm{a}}$ & & Mean (SD) & $3.5(4.7)$ & $3.1(3.0)$ \\
\hline $\mathrm{Ca} 2+(\mathrm{mmol} / \mathrm{L})^{\mathrm{a}}$ & & Mean (SD) & $2.36(0.15)$ & $2.34(0.11)$ \\
\hline
\end{tabular}

${ }^{\text {a }} p$ value based on a two-sample $t$ test

${ }^{\mathrm{b}} p$ value based on a Mann-Whitney test

* $p$ value based on a Fisher's exact test 
participants reported osteoporosis in their medical history, similar in both groups. Intervention compliance was high (median: 93\%) from baseline to follow-up, and did not differ between treatment groups. Body weight increased equally and significantly (by $\sim 1 \mathrm{~kg}$ ) over the 13-week intervention in both active and control treatment groups [data not shown] [14].

Total body BMD increased in the active group only, and the improvement was significantly different from control $(p=0.033)$ (Table 2). Serum 25(OH)D concentrations increased on average by $26 \mathrm{nmol} / \mathrm{L}(p<0.001)$ in the active group and decreased on average by $6 \mathrm{nmol} / \mathrm{L}$ in the control group after 13 weeks (Table 2). The prevalence of serum $25(\mathrm{OH}) \mathrm{D}$ concentration below $50 \mathrm{nmol} / \mathrm{L}$ decreased from 53 to $8 \%$ in the active group, whilst the proportion of those in the control group with serum $25(\mathrm{OH}) \mathrm{D}$ below $50 \mathrm{nmol} / \mathrm{L}$ did not decrease during the study (51\% at baseline and $66 \%$ at week 13). Serum PTH concentrations showed significant treatment differences $(p<0.001)$ with decreased concentrations in the active treatment group and increased concentrations in the control group after 13 weeks (Table 2). Changes in serum CTX concentrations were significantly different
Table 2 Biochemical measures of vitamin D status, bone turnover, IGF-1 and BMD among treatment groups before and after intervention

\begin{tabular}{|c|c|c|c|c|}
\hline & \multicolumn{4}{|l|}{ Treatment group } \\
\hline & Control $n=196$ & Active $n=184$ & $\begin{array}{l}p \text {-value for change, } \\
\text { active versus control }^{\mathrm{a}}\end{array}$ & $\begin{array}{l}p \text {-value } \\
\text { further } \\
\text { adjusted }^{\text {b }}\end{array}$ \\
\hline \multicolumn{5}{|c|}{ Serum 25(OH)D (nmol/L) } \\
\hline Pre-intervention ${ }^{c}$ & $52.01(23.03)$ & $51.05(22.86)$ & & \\
\hline Post-intervention $^{\mathrm{d}}$ & $46.06(21.40)$ & $78.89(21.06)$ & $<.001^{\mathrm{e}}$ & $<.001^{\mathrm{e}}$ \\
\hline \multicolumn{5}{|l|}{ Serum PTH (pmol/L) } \\
\hline Pre-intervention ${ }^{\mathrm{c}}$ & $6.75(4.67)$ & $6.82(4.05)$ & & \\
\hline Post-intervention ${ }^{\mathrm{d}}$ & $7.30(5.07)$ & $5.39(2.38)$ & $<.001^{\mathrm{e}}$ & $<.001^{\mathrm{e}}$ \\
\hline \multicolumn{5}{|l|}{ Serum OC $(\mu \mathrm{g} / \mathrm{L})$} \\
\hline Pre-intervention ${ }^{\mathrm{c}}$ & $4.51(3.79)$ & $4.63(3.67)$ & & \\
\hline Post-intervention $^{\mathrm{d}}$ & $5.27(4.50)$ & $4.65(3.65)$ & $0.108^{\mathrm{e}}$ & $0.099^{\mathrm{e}}$ \\
\hline \multicolumn{5}{|l|}{ Serum P1NP (ng/ml) } \\
\hline Pre-intervention $^{c}$ & $52.44(33.14)$ & 49.67 (27.39) & & \\
\hline Post-intervention $^{\mathrm{d}}$ & $54.53(44.95)$ & $50.63(27.85)$ & 0.096 & 0.119 \\
\hline \multicolumn{5}{|l|}{ Serum CTx (ng/ml) } \\
\hline Pre-intervention ${ }^{c}$ & $0.29(0.20)$ & $0.28(0.22)$ & & \\
\hline Post-intervention $^{\mathrm{d}}$ & $0.27(0.25)$ & $0.24(0.23)$ & $<.001$ & 0.001 \\
\hline \multicolumn{5}{|l|}{ Serum IGF-1 $(\mu \mathrm{g} / \mathrm{L})$} \\
\hline Pre-intervention ${ }^{\mathrm{c}}$ & $124.03(73.28)$ & $113.99(42.55)$ & & \\
\hline Post-intervention $^{\mathrm{d}}$ & $119.40(75.00)$ & $126.46(48.94)$ & $<.001$ & $<.001$ \\
\hline \multicolumn{5}{|c|}{ Serum Ca (corrected for albumin) } \\
\hline Pre-intervention ${ }^{c}$ & $2.31(0.15)$ & $2.29(0.12)$ & & \\
\hline Post-intervention $^{\mathrm{d}}$ & $2.31(0.16)$ & $2.33(0.13)$ & $<.001$ & $<.001$ \\
\hline \multicolumn{5}{|c|}{ Bone mineral density $\left(\mathrm{g} / \mathrm{cm}^{2}\right)$} \\
\hline Pre-intervention ${ }^{c}$ & $1.07(0.19)$ & $1.07(0.19)$ & & \\
\hline Post-intervention $^{\mathrm{d}}$ & $1.07(0.23)$ & $1.09(0.18)$ & 0.033 & 0.033 \\
\hline
\end{tabular}

Values represent means and standard deviations

PTH parathyroid hormone, 25(OH)D 25-hydroxyvitamin D, OC osteocalcin, CTx carboxyterminal crosslinked telopeptide of type 1 collagen

${ }^{a}$ The change from pre- to post-intervention values was compared between the active and control group using a two-sample $t$ test (equal variances assumed)

${ }^{\mathrm{b}}$ Analysis of covariance which controlled for pre-intervention concentration of the variable as well as age and sex with a random effect for site (small sites combined)

${ }^{\mathrm{c}}$ Baseline measurement (measurement at screening for subjects with no baseline data), prior to first intake of the investigational product

${ }^{\mathrm{d}}$ Measurement after 13 weeks of intake of the investigational product

${ }^{\mathrm{e}}$ The data were log transformed for the statistical tests to obtain a better approximation of the normal distribution based on visual inspection of the residuals of the ANCOVA models 
$(p<0.001)$ between the two interventions, with lower values in both treatment groups after 13 weeks, but the decline was more marked in the active treatment group. Serum calcium (corrected for albumin) increased slightly after 13 weeks in the active treatment group only ( $p<0.001$; Table 2$)$. Serum IGF-1 change during intervention was significantly different between active and control groups $(p<0.001$; Table 2$)$ with decreased concentrations in the control group and significantly increased concentrations in the active group. There were no statistical significant treatment differences in change from pre- to post-intervention values in serum OC and P1NP. Analyses corrected for age, sex and study site showed very similar and consistent results (Table 2).

\section{Discussion}

The findings of this 13-week nutritional intervention among non-malnourished older sarcopenic adults showed important improvements in vitamin D status (by 55\%), decreased PTH (by $21 \%$ ) and increased IGF-1 (by $11 \%$ ) concentrations in those participants who consumed the vitamin D (1600 IU/ day), calcium (1000 mg/day), and leucine-enriched whey protein ( $40 \mathrm{~g} /$ day whey protein and $6 \mathrm{~g} /$ day total leucine) supplement. In addition, concentrations of the bone resorption marker CTX declined in both treatment groups although the decline was more pronounced in the active group. We postulate that the ***reduction in CTX in the control group [and to some degree in the active treatment group] might be explained by an increase in energy intake (and thus energy availability) over the 13 weeks in the participants.

Mean daily energy intake increased by $166 \mathrm{kcal} /$ day and $165 \mathrm{kcal} /$ day during the 13 weeks intervention period in the active and control groups, respectively [14]. A dose-response inverse relationship between energy availability and bone resorption has been demonstrated in young adult women [20] and dietary energy restriction leads to raised bone resorption in post-menopausal women [21]. Validating our assumption is the fact that participants on control and active treatment gained approximately $1 \mathrm{~kg}$ during the intervention suggesting a net positive energy balance in the study participants. Circulating concentrations of bone formation markers OC and P1NP did not change in either group during the intervention. Despite the lack of change in bone formation markers with active treatment, the observed large increase in circulating $25(\mathrm{OH}) \mathrm{D}(>30 \mathrm{nmol} / \mathrm{l})$ and concomitant reduction in PTH concentration in those participants receiving active treatment may have potential benefits to bone health. Indeed, consumption of the active product showed small but significant improvements in total body BMD after 13 weeks of treatment in comparison to those on the control product. However, whether the observed changes in total body BMD are transient or sustained after supplementation remains unclear. The observed difference in IGF-1 concentrations between the treatment groups may also offer some explanation as to why those receiving active treatment had significantly higher BMD after the intervention. It is known that IGF-1 regulates key signalling pathways in bone including those involved in cell survival, protein synthesis and energy utilization [22], thus potentially mediating the apparent positive effect of protein supplementation on BMD. Although serum calcium adjusted for albumin was lower in the active treatment group at baseline, a small statistically significant increase was observed in those participants on active treatment after 13 weeks. All serum calcium values remained within the normal range on the 13-weeks supplementation. Moreover, no (serious) adverse events point at hypercalcemia were recorded during the study.

Similar nutritional interventions in older adults support combined protein and vitamin D/calcium supplementation in ameliorating bone turnover but in particular bone resorption. In a study of 89 vitamin $\mathrm{D}$ deficient institutionalized women $\geq 60$ years old living in 10 French nursing homes, Bonjour et al. [23] observed a $20 \%$ decrease in PTH and an $8 \%$ decrease in CTX after consumption of a vitamin D (400 IU) and calcium-fortified $(800 \mathrm{mg})$ yoghurt twice daily, over 56 days. These effects are similar to the active intervention in our study that resulted in a $21 \%$ and $14 \%$ decrease of PTH and CTX, respectively. A significant decline in PTH and bone resorption markers was also observed after 84 days of vitamin D (400 IU) and calcium-fortified ( $800 \mathrm{mg}$ ) yoghurt consumption twice daily in a community-dwelling population of 57 women aged $>60$ years, (mean age 73 years, BMI $30 \mathrm{~kg}$ / $\mathrm{m}^{2}$ ) living in a sheltered accommodation housing in Hull (England) [24]. Similar effects of calcium and/or vitamin D fortified milk on bone parameters have been observed in Asian populations. A 16-weeks calcium or calcium/ vitamin D fortified milk trial in post-menopausal Asian women demonstrated reductions in PTH and bone turnover of about $10 \%$ and $\geq 20 \%$ respectively $[25,26]$. Two longer term intervention studies with a duration between 18 and 30 months using vitamin D fortified dairy with or without calcium have shown beneficial effects on bone turnover and/or total and site-specific BMD in postmenopausal women [26, 27]. In an 18-month placebo-controlled randomized intervention in post-menopausal Chinese women receiving calcium-fortified milk, providing $900 \mathrm{mg}$ calcium and 256 IU vitamin D daily, BMD at the hip was improved by $\sim 2 \%$ with treatment, but no change was observed at the lumbar spine [26]. Total body BMD was maintained in 50 post-menopausal women over 30 months when provided calcium/vitamin D fortified dairy foods $(\sim 1200 \mathrm{mg}$ calcium and 300 IU vitamin D) daily, relative to a decline in BMD in the control group $(0.3 \%$ vs. $-1.8 \%)$ [27]. These alterations in BMD are comparable to those observed 
in our study, i.e. $\sim 2 \%$ increase in BMD. One possibility which may explain the improvement in BMD observed in the active treatment group after 13 weeks in our study is the concomitant improvement seen in IGF-1 concentrations after protein supplementation. IGF-1 is a growth-promoting protein that plays essential roles in growth and development including influencing skeletal growth in all stages of life. It is well known that the secretion IGF-1 declines during adulthood to older age until only low levels are detected in adults $\geq 60$ years however, the biological significance of this decline is unclear. Whilst the precise mechanism by which circulating IGF-1 functions to maintain bone mass is not fully understood, evidence suggests that circulating IGF-1 is strongly and positively associated with BMD at several sites in older women from the Framingham cohort [28]. Moreover, IGF-1 can also be considered as the cross road of the nutritional, inflammatory and hormonal pathways in the frailty process [29] and one of the main actors of the cross-talk between bone and muscle [4].

The improvement in total body BMD in this study compliments the improvements previously observed in muscle mass and lower-extremity function in the same study [14], and adds further support to the hypothesis that osteopenia and sarcopenia are inextricably linked [30]. Moreover, the observations in this study may offer a potential benefit for older people at risk of fragility fractures. The 13-weeks intervention phase in this study, although sufficient to observe changes in bone resorption markers, may not have been long enough to detect corresponding changes in bone formation. In addition, we cannot conclude whether the beneficial intervention effects on bone are due to high protein, high leucine, vitamin $\mathrm{D}$, calcium and/or other micronutrients in the active treatment such as magnesium and phosphorus which have a well-established role in bone metabolism or indeed whether there is a 'synergistic effect' due to the combination of bone related nutrients in the active product. Despite the relatively high phosphorus content of the supplement it is our view that given the likelihood of suboptimal dietary intakes of vitamin D and protein in these participants [and the general geriatric population] the positive effects on vitamin D status, $\mathrm{PTH}$, IGF-1 and BMD observed in the active treatment group is most likely due to a combination of vitamin $\mathrm{D}$ and protein supplementation. Analysing the nutritional intake of a subset of the PROVIDE study participants [31], it appeared that the dietary intake of phosphorus was sufficient (mean intakes $=1196 \mathrm{mg} / \mathrm{day}$, which are well above current reference intakes) suggesting that additional phosphorus to already replete phosphorus diets may not be a large contributor to the positive effects on bone health in this study.

In conclusion, consuming a vitamin $\mathrm{D}$, calcium and leucine- enriched whey protein supplement for 13 weeks improved vitamin D status, suppressed serum PTH and had small but significant effects on BMD in sarcopenic non-malnourished older adults. This study shows proofof-principle that a specific nutritional intervention alone might benefit vitamin D status and bone health in older adults.

Acknowledgements We are grateful to Fatma Gossiel from the University of Sheffield for undertaking the bone turnover marker analysis. We would like to thank Dr Yvette Luiking from Danone Medical Nutrition for the helpful comments on the manuscript.

Funding The study was funded by Danone Nutricia Research, Nutricia Advanced Medical Nutrition.

\section{Compliance with Ethical Standards}

Conflict of interest Richard Eastell consulted for and has had research funding from Roche Diagnostics. Tom Hill has received honoraria from the Dairy Council (GB). Sjors Verlaan, Egbert Biesheuvel, Jürgen M. Bauer, Ivan Bautmans, Kirsten Brandt, Lorenzo M. Donini, Marcello Maggio, Tony Mets, Chris J Seal, Sander LJ Wijers, Cornel Sieber, Tommy Cederholm \& Terry J. Aspray declare that they have no conflict of interest.

Ethical Approval The Research Ethics Boards at each of the study locations reviewed and approved the study protocol. The trial was registered in the Dutch trials register with the identifier: NTR2329 (http:// www.trialregister.nl/trialreg) and was conducted in accordance with the Declaration of Helsinki.

Informed Consent All procedures followed were in accordance with the ethical standards of the responsible committee on human experimentation (national and institutional). Informed consent was obtained from all individual subjects participating in the study.

Open Access This article is distributed under the terms of the Creative Commons Attribution 4.0 International License (http://creativeco mmons.org/licenses/by/4.0/), which permits unrestricted use, distribution, and reproduction in any medium, provided you give appropriate credit to the original author(s) and the source, provide a link to the Creative Commons license, and indicate if changes were made.

\section{References}

1. Cruz-Jentoft AJ, Baeyens JP, Bauer JM et al (2010) Sarcopenia: European consensus on definition and diagnosis: report of the European working group on Sarcopenia in older people. Age Ageing 39(4):412-423

2. Rizzoli R, Stevenson JC, Bauer JM et al (2014) The role of dietary protein and vitamin $\mathrm{D}$ in maintaining musculoskeletal health in postmenopausal women: a consensus statement from the European Society for Clinical and Economic Aspects of Osteoporosis and Osteoarthritis (ESCEO) ESCEO Task Force. Maturitas 79(1):122-132

3. Huo YR, Suriyaarachchi P, Gomez F et al (2015) Phenotype of osteosarcopenia in older individuals with a history of falling. $\mathrm{J}$ Am Dir Assoc 16(4):290-295 
4. Tagliaferri C, Wittrant Y, Davicco MJ, Walrand S, Coxam V (2015) Muscle and bone, two interconnected tissues. Ageing Res Rev 21:55-70

5. Rizzoli R, Stevenson JC, Bauer JM et al (2014) The role of dietary protein and vitamin $\mathrm{D}$ in maintaining musculoskeletal health in postmenopausal women: a consensus statement from the European Society for Clinical and Economic Aspects of Osteoporosis and Osteoarthritis (ESCEO). ESCEO Task Force. Maturitas 2079(1):122-132

6. Schurch MA, Rizzoli R, Slosman D et al (1998) Protein supplements increase serum insulin-like growth factor-I levels and attenuate proximal femur bone loss in patients with recent hip fracture. A randomized, double-blind, placebo-controlled trial. Ann Intern Med. 128(10):801-809

7. Gaffney-Stomberg E, Insogna KL, Rodriguez NR, Kerstetter JE (2009) Increasing dietary protein requirements in elderly people for optimal muscle and bone health. J Am Geriatr Soc 57(6):1073-1079

8. Garnero P, Sornay-Rendu E, Delmas PD (2000) Low serum IGF-1 and occurrence of osteoporotic fractures in postmenopausal women. Lancet 355(9207):898-899

9. Shams-White MM, Chung M, Du M et al (2017) Dietary protein and bone health: a systematic review and meta-analysis from the National Osteoporosis Foundation. Am J Clin Nutr 105(6): 1528-1543

10. Jennings A, MacGregor A, Spector T, Cassidy A (2016) Amino acid intakes are associated with bone mineral density and prevalence of low bone mass in women: evidence from discordant monozygotic twins. J Bone Min Res 31(2):326-335

11. Ammann P, Laib A, Bonjour JP et al (2002) Dietary essential amino acid supplements increase bone strength by influencing bone mass and bone microarchitecture in ovariectomized adult rats fed an isocaloric low-protein diet. J Bone Min Res 17(7):1264-1272

12. Bauer J, Biolo G, Cederholm T, Cesari M, Cruz-Jentoft AJ, Morley JE et al (2013) Evidence-based recommendations for optimal dietary protein intake in older people: a position paper from the prot-age study group. J Am Med Dir Assoc 4(8):542-559

13. Ross C, Abrams S, Aloia JF et al (2010) Institute of Medicine. Dietary Reference Intakes for Calcium and Vitamin D, Washington, USA

14. Bauer JM, Verlaan S, Bautmans I et al (2015) Effects of a vitamin $\mathrm{D}$ and leucine-enriched whey protein nutritional supplement on measures of sarcopenia in older adults, the PROVIDE study: a randomized, double-blind, placebo-controlled trial. J Am Med Dir Assoc 16(9):740-747

15. Verlaan S, Maier AB, Bauer JM et al (2018) Sufficient levels of 25-hydroxyvitamin $\mathrm{D}$ and protein intake required to increase muscle mass in sarcopenic older adults-The PROVIDE study. Clin Nutr 37(2):551-557

16. Scafoglieri A, Clarys JP, Bauer JM et al (2017) Predicting appendicular lean and fat mass with bioelectrical impedance analysis in older adults with physical function decline-The PROVIDE study. Clin Nutr 36:869-875

17. Sergi G, De Rui M, Veronese N et al (2015) Assessing appendicular skeletal muscle mass with bioelectrical impedance analysis in free-living Caucasian older adults. Clin Nutr 34:667-673
18. Paddon-Jones D, Rasmussen BB (2009) Dietary protein recommendations and the prevention of sarcopenia. Curr Opin Clin Nutr Metab Care 12(1):86-90

19. Scafoglieri A, Provyn S, Wallace J et al (2011) Whole body composition by hologic QDR 4500/A DXA: system reliability versus user accuracy and precision. In: Ivanoc $\mathrm{O}$ (ed) Applications and experiences of quality control. InTech, Croatia

20. Ihle R, Loucks AB (2004) Dose-response relationships between energy availability and bone turnover in young exercising women. J Bone Min Res 19(8):1231-1240

21. Ricci TA, Heymsfield SB, Pierson RN Jr et al (2001) Moderate energy restriction increases bone resorption in obese postmenopausal women. Am J Clin Nutr 73(2):347-352

22. Guntur AR, Rosen CJ (2013) IGF-1 regulation of key signaling pathways in bone. Bone key Rep 2:437

23. Bonjour JP, Benoit V, Payen F, Kraenzlin M (2013) Consumption of yogurts fortified in vitamin $\mathrm{D}$ and calcium reduces serum parathyroid hormone and markers of bone resorption: a double-blind randomized controlled trial in institutionalized elderly women. $\mathrm{J}$ Clin Endocrinol Metab 98(7):2915-2921

24. Bonjour JP, Benoit V, Atkin S, Walrand S (2015) Fortification of yogurts with vitamin $\mathrm{D}$ and calcium enhances the inhibition of serum parathyroid hormone and bone resorption markers: a double blind randomized controlled trial in women over 60 living in a community dwelling home. J Nutr Health Aging 19(5):563-569

25. Kruger MC, Schollum LM, Kuhn-Sherlock B et al (2010) The effect of a fortified milk drink on vitamin D status and bone turnover in post-menopausal women from South East Asia. Bone 46(3):759-767

26. Gui JC, Brasic JR, Liu XD et al (2012) Bone mineral density in postmenopausal Chinese women treated with calcium fortification in soymilk and cow's milk. Osteoporos Int 23(5):1563-1570

27. Moschonis G, Katsaroli I, Lyritis GP, Manios Y (2010) The effects of a 30-month dietary intervention on bone mineral density: the postmenopausal health study. Br J Nutr 104(1):100-107

28. Langlois JA, Rosen CJ, Visser M et al (1998) Association between insulin-like growth factor I and bone mineral density in older women and men: the Framingham heart study. J Clin Endocrinol Metab 83:4257-4262

29. Maggio M, De Vita F, Lauretani F et al (2013) IGF-1, the cross road of the nutritional, inflammatory and hormonal pathways to frailty. Nutrients 5(10):4184-4205

30. Drey M, Sieber CC, Bertsch T, Bauer JM, Schmidmaier R (2016) The FiAT intervention group. Osteosarcopenia is more than sarcopenia and osteopenia alone. Ageing Clin Exp Res 28:895-899

31. Verlaan S, Aspray TJ, Bauer J et al (2017) Nutritional status, body composition, and quality of life in community dwelling sarcopenic and non-sarcopenic older adults: a case control study. Clin Nutr $36: 267-274$

Publisher's Note Springer Nature remains neutral with regard to jurisdictional claims in published maps and institutional affiliations. 


\section{Affiliations}

\section{Tom R. Hill ${ }^{1}\left[\right.$ - Sjors Verlaan ${ }^{2,5}$ • Egbert Biesheuvel ${ }^{2} \cdot$ Richard Eastell $^{3}$. Jürgen M. Bauer ${ }^{6}$. Ivan Bautmans ${ }^{7}$. Kirsten Brandt ${ }^{1}$. Lorenzo M. Donini ${ }^{8} \cdot$ Marcello Maggio $^{9} \cdot$ Tony Mets $^{7}$. Chris J. Seal ${ }^{1}$. Sander LJ Wijers ${ }^{2}$. Cornel Sieber ${ }^{10}$. Tommy Cederholm ${ }^{11}$. Terry J. Aspray ${ }^{4}$ on behalf of the PROVIDE Consortium}

1 Institute of Cellular Medicine, Human Nutrition Research Centre, Newcastle University, Newcastle upon Tyne NE2 4HH, UK

2 Danone Nutricia Research, Nutricia Advanced Medical Nutrition, Uppsalalaan 12, Utrecht Science Park, Utrecht 3584 CT, The Netherlands

3 Academic Unit of Bone Metabolism, Metabolic Bone Centre, Northern General Hospital, University of Sheffield, Sheffield S5 7AU, UK

4 Bone Clinic, Institute of Cellular Medicine, Freeman Hospital, Newcastle University, Newcastle upon Tyne NE7 7DE, UK

5 Department of Internal Medicine, Section of Gerontology and Geriatrics, Amsterdam UMC, Amsterdam, The Netherlands

6 Department of Geriatric Medicine, Carl von Ossietzky University, Oldenburg, Germany
7 Frailty in Ageing Research Group (FRIA), Vrije Universiteit Brussel (VUB), Brussels, Belgium

8 Department of Experimental Medicine, Section of Medical Pathophysiology, Endocrinology and Human Nutrition, "Sapienza" University of Rome, Rome, Italy

9 Department of Clinical and Experimental Medicine, Section of Geriatrics, University of Parma, Parma, Italy

10 Institute for Biomedicine on Ageing, Friedrich-Alexander-University Erlangen-Nürnberg, Erlangen, Germany

11 Department of Public Health and Caring Sciences/Clinical Nutrition and Metabolism, Department of Geriatric Medicine, Uppsala University Hospital, Uppsala, Sweden 